\title{
Toxicity Bioassays of Synthetic Dyes Biosorbed by Heat-Inactivated Lentinus Edodes Ccb-42 Immobilized in Loofa Sponges
}

\author{
Gabriela Gregolin Gimenez (I), Suelen Pereira Ruiz (I), Graciette \\ Matioli (I) \\ (I) UEM - Universidade Estadual de Maringá (Av. Colombo , 5.790. CEP: 87020-900, Maringá, \\ $\mathrm{PR}, \mathrm{BR})$
}

\section{Resumo}

It is difficult to treat wastewater containing dyes and a wide variety of synthetic dyes have been applied in many industries. Bordeaux red is a synthetic dye used in food. In this study heat-inactivated Lentinus edodes CCB-42 was immobilized in loofa sponges and applied to the biosorption of bordeaux red, and toxicity bioassay of the dye-containing medium was taken before and after treatment. The biosorption conditions were optimized and it was possible to remove $80.8 \%$ of bordeaux red using heatinactivated L. edodes. Toxicity tests were performed to determine whether treatment with heat inactivated L. edodes reduced the toxicity of the dye solutions. Buffer solutions with $200 \mathrm{mg} / \mathrm{l}$ dye, called raw effluent, were tested along with the solution treated with inactivated and immobilized microorganism under ideal conditions, called treated effluent. Phytotoxicity tests were conducted using Lactuca sativa Aurelia seeds and raw and treated effluent samples were diluted to 1, 3, 10, 30 and 100\% using hard reconstituted water. Acute ecotoxicity tests were performed according to the methodology of solution cultivation for hatching Artemia salina cysts and samples of raw and treated effluents were diluted to yield solutions with 20, 40, 60, 80 and $100 \%$ effluent. The percentage of germination was higher in more dilute samples, and no germination occurred in samples containing $100 \%$ wastewater. In all cases, the germination percentage of $\mathrm{L}$. sativa seeds was higher in the treated effluent after biosorption by heat- 
inactivated L. edodes immobilized in loofa sponge. While this species was able to partially grow in potentially toxic medium, lethal effects (inhibition of germination) and sub-lethal effects (growth inhibition of roots and radicles) were observed. The median lethal dose (LD50) values for raw and treated effluents using the species Lactuca sativa was $10,42 \%$ and $16,71 \%$, respectively. The acute ecotoxicity was tested with micro crustaceous Artemia salina . After $24 \mathrm{~h}$ of incubation, no surviving organisms were found on any of the tubes analyzed. The toxicity to Artemia salina demonstrated that this species was not able to develop in samples of the raw and treated effluent, regardless of the dilution. The reduced phytotoxicity of the dye solution following treatment, strengthens the potential applicability of L. edodes CCB-42 in the biosorption of synthetic dyes.

Palavras-Chave: Lentinus edodes, bordeaux red, biosorption, phytotoxicity, acute ecotoxicity

Agência de Fomento: We thank the Brazilian agencies CAPES, CNPq and Fundação Araucaria for financial support and the company Duas Rodas, Santa Catarina, for providing the bordeaux red dye. 